Journal of Economics and Behavioral Studies (ISSN: 2220-6140)

Vol. 8, No. 4, pp. 32-40, August 2016

\title{
Fertility Status of Married Women and Its Determinants in Ethiopia
}

\author{
Essey Kebede Muluneh ${ }^{* 1}$, Temesgen Zewotir2, Tilaye Matebe Yayeh ${ }^{3}$ \\ ${ }^{1}$ Bahir Dar University, Bahir Dar, Ethiopia \\ ${ }^{2}$ University of Kwazulu Natal, South Africa \\ ${ }^{3}$ Wollo University, Dessie, Ethiopia \\ *kebedeessey@yahoo.com
}

\begin{abstract}
This study investigates determinant factors of fertility among married women in Ethiopia, the second most populous country in Africa with rapid population growth. The data used for the analysis was obtained from the 2014 Ethiopia Mini Demographic and Health Survey which was carried out by the Central Statistical Agency. A generalized linear model (GLM) analysis was carried out to investigate the effect of socioeconomic and demographic factors on the number of children ever born by a married woman of age 1549 years. High fertility was independently associated with residing in urban areas, increased household economic status, younger age at first birth and not using contraceptives. Current age and media exposure, household head gender and media exposure, household head gender and regional state, mother's education and, regional state and media exposure and regional state were found to jointly affect fertility level.
\end{abstract}

Keywords: Fertility status; children ever born; generalized linear model; Poisson regression; negative binomial regression; rate ratios

\section{Introduction}

Ethiopia is the second most populous country in Africa with a total population of 98.1 million by mid-2015 and projected population of 130.5 million and 165.1 million by mid-2030 and mid-2050 respectively (Population Reference Bureau, 2015). This rapid population growth is partly due to a relatively high fertility rate. The 2014 Ethiopia Mini Demographic and Health Survey reported that in Ethiopia total fertility rate (TFR) for the three years preceding the survey was 4.1 children per women. The report also showed that fertility declined between 2005 and 2011, from 5.4 children per woman to 4.8, and then decreased further to 4.1 children in 2014 (CSA, 2014). Even though the total fertility rate of Ethiopia is less than that for the SubSaharan region (=5.0), it still high like many of African countries as compared to the world average of 2.5 (Population Reference Bureau 2015). The 2005 report of the Department of Economic and Social Affairs of the United Nations (UN, 2005) also rated the fertility rate of the country as one of the top nine countries in terms of population increase from 2000-2050. The social, economic and cultural setups in rural parts of Ethiopia are still in favor of having large number of children. This partly results from the value the society gives to children, preferences relating to the sex of a child, economic needs and old age security. High fertility however has adverse effects on the health of the child and the mother and child schooling. In countries like Ethiopia where the livelihood of about eighty five percent of the population depends on agricultural practices on small individual holdings, continuous population growth may result in environmental degradation which ultimately contributes to global warming.

Identifying factors that determine household's ideal or preferred number of children and designing strategies that affect fertility choices requires comprehensive studies. A number of descriptive and informative studies on the households' ideal or preferred number of children were conducted in Ethiopia (Hailemariam, 1992; Falls, 2007; Teklu, Sebhatu and Gebreselassie, 2013; Alemayehu, Haider and Habte, 2010; Jara, Dejene and Taha, 2013; Gebremedhin and Betre, 2009). These studies have greatly contributed to our understanding of many aspects of determinants of fertility in Ethiopia. However, these studies are either limited to urban population or focused more on descriptive statistical analyses. But advanced statistical analysis is useful to measure the intrinsic and explicit effects of the socio-economic, demographic and environmental factors. Accordingly this study is aimed to identify socio economic and demographic determinant factors of fertility 
among married women in Ethiopia. The study employed generalized linear model for in-depth investigation of the joint effect of two or more risk factors.

The Data: The data used for the analysis was obtained from the 2014 Ethiopia Mini Demographic and Health Survey EDHS which was carried out by Central Statistical Agency (CSA). The main objective of the survey was to collect population-based data on key demographic, socio-economic and health indicators that support the monitoring and evaluation health sector development program. The survey questionnaire were used; one for the household and one for women. A total of 8475 households were interviewed. The age and gender of household members obtained in the household questionnaire were used to identify women who were eligible for the individual interview. The women's questionnaire was used to collect information from 4617 married women in the childbearing age 15-49. These women were asked on the number of children ever born. The respondents were asked questions with regards to their current age, education, age at first birth, birth history, contraceptive use and media exposure. Besides these characteristics, the wealth index based on the household assets and sanitation facilities was automated in the survey database.

Ethiopia is constitutionally formed as a federation of nine ethnically-based regional states and two chartered cities. These eleven administrative regions have basic roles of managing public health services and coordinating the operation of health system under their jurisdiction. Regional states therefore account such cultural and administrative diversity. Likewise the difference in rural and urban mindsets is assessed by including rural-urban classification of the respondents. The frequency distribution of the number of children ever born is presented Figure 1. As can be seen, the distribution is highly skewed with the low number of children ever born as the modal and median responses. The median and mean number of children ever born per married woman is 4.0 and 4.2 respectively.

\section{Figure 1: The Percentage of Married Women by the Number of Children Ever Born}

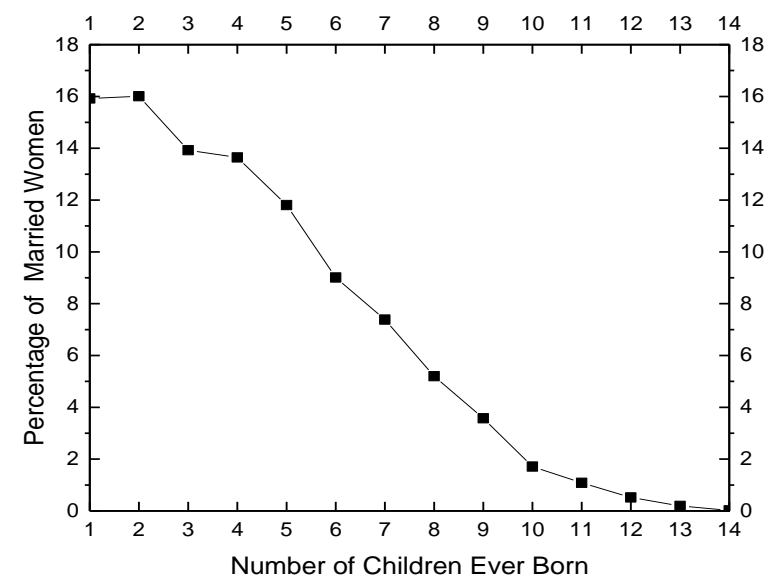

\section{Methodology}

A generalized linear model (GLM) analysis was carried out to investigate the demographic, socio-economic and geographic factors affecting respondents' number of living children ever born. Since the response variable of interest, which is the total number of children ever born, is a count data, it may not be reasonable to assume that the data were normally distributed. As a result the traditional linear regression model is not applicable. A GLM extends the traditional linear model to a wider range of data analysis problems (log normal, inverse Gaussian, gamma, Poisson, binomial, negative binomial) and a function can be used to link the expected value (mean) of the response and a linear function of the explanatory variables. In short, a GLM can be constructed by choosing an appropriate link function and response probability distribution (Agresti, 2002). 
The best known GLMs for count responses assume a Poisson or a negative binomial distribution. The Poisson regression restricts the response variable to have mean-variance equality. If this assumption is violated, the resulting estimates are consistent, but, estimates of the variance are not. This can result in spuriously small standard errors of the estimates (Barron, 1992). In such over or under dispersion scenario, from statistical viewpoint, Poisson distribution might not be acceptable. A common way to deal with overcomes the problem of over and under-dispersion for counts are to use the use of "quasi-likelihood" in a generalized linear model framework (McCullagh and Nelder, 1989). The quasi-Poisson regression provides valid inference, guarding against drawing of incorrect conclusions (see Allison, 2001; Ver Hoef and Boveng, 2007 and Zewotir and North, 2015). The interpretation of the parameter estimates in the quasi-likelihood approach remains as ordinary Poisson regression. Indeed we are aware that the alternative over-dispersion remedy is the negative binomial regression (Zewotir and Ramroop, 2009 and Lindén and Mäntyniemi, 2011). For any given data set, information theoretic approaches such as Akaike information or Bayesian information criteria might be considered to choose between a quasi-Poisson model and a negative binomial. However, a good understanding of the theoretical differences between them can form the basis for an a priori decision based on scientific purposes, which the quasi model formulation has the advantage of leaving parameters in a natural, interpretable state and allows standard model diagnostics without a loss of efficient fitting algorithms (Ver Hoef and Boveng, 2007). In other words, the quasi Poisson strategy leads to the same coefficient estimates as the standard Poisson model but inference is adjusted for over-dispersion. If overdispersion is not the issue the Poisson distribution is the natural distribution of the count data.

A generalized linear model (GLM) analysis was carried out to investigate the effect of current age, education, and age at first birth, birth history, and contraceptive use, and media exposure, gender of household head, household wealth, regional state and residential area on the number of children ever born by a married woman. It is reasonable to assess the magnitude of the effect of several factors acting jointly over and above their effects considered separately. In other words, the extent to which the effect of one factor changes for different values of one or more other factors needs to be measured, this is called the interactions effect. The significance of the interaction effects were looked at by adding them into the main effects model one at a time and retaining the significant interactions. All the three-way and higher-level interactions effects were obtained non-significant. Accordingly, the final model was found to be the main effects and five two-way interactions. Media exposure interacted with three variables current age of the mother, gender of the household head and regional state; the other two interactions were the household head gender and regional state, and regional state and educational level of the mother. For this model we first examined the Pearson Chi-square or deviance divided by the degrees of freedom for over/under dispersion. Values greater than 1 indicate over-dispersion, that is, the true variance of the number of children ever born by married woman is greater than the expected number of children ever born per woman, under the given model. From Table 1, the values are sufficiently close to 1 and indicated no evidence of over-dispersion and the adequacy of the Poisson regression fit.

Table 1: Assessment of Over/Under Dispersion in the Poisson Regression

\begin{tabular}{lccl}
\hline \multicolumn{3}{c}{ Log Link } & \\
Criterion & DF & Value & Value/DF \\
\hline Deviance & & 4800.74 & 1.06 \\
Pearson Chi-Square & 4544 & 4766.91 & 1.05 \\
\hline
\end{tabular}

We further compared the goodness of fit of Poisson regression with the quasi Poisson and negative binomial regressions (refer Table 2). The AIC and corrected AIC (AICC) slightly favor the goodness of negative binomial and BIC favors Poisson regression by the same margin. Notably these approaches depend on a distributional form and a likelihood and the comparison between Poisson and negative binomial distributions was valid; however, quasi models are only characterized by their mean and variance, and do not necessarily have a distributional form. Quasi-AIC (QAIC) might be appropriate, but QAIC is useful within the quasi class of models and not between quasi models and models with distributional forms. Since the variance to mean ratio is roughly 1 , (in fact 1.06 or 1.05 , refer Table 1) the quasi-Poisson adjustment fitness is the same as the Poisson regression and that result was demonstrated in Table 2. All the avenues indicated that the impact of using negative binomial and/or quasi-Poisson to overcome over dispersion in this Poisson regression would be worthless. 
Table 2: Model Selection among Poisson, Quasi-Poisson and Negative Binomial Regressions

\begin{tabular}{lccc}
\hline $\begin{array}{l}\text { Criteria: Smaller is } \\
\text { better }\end{array}$ & Poisson & $\begin{array}{c}\text { Regression } \\
\text { Quasi-Poisson }\end{array}$ & Negative Binomial \\
\hline AIC & 19349.50 & 19349.50 & 19346.13 \\
AICC & 19351.88 & 19351.88 & 19348.57 \\
BIC & 19819.44 & 19819.44 & 19822.50 \\
\hline
\end{tabular}

\section{Results}

A set of model diagnostics was performed before any inference from the model. The index plots for the residuals and the deviance residuals of the selected model showed that there were no observations that were poorly accounted for by the model. The index plot of the diagonal elements of the hat matrix suggests that there are no extreme points in the design space that one needs to consider. Furthermore, an index plot of Cook's distance indicated that there are no observations that may have a large impact on the estimated coefficients and thus on the goodness of fit of the model. The diagnostics results in Figure 2 allow us to make statistical tests and inferences.

Figure 2: Model diagnostics plots

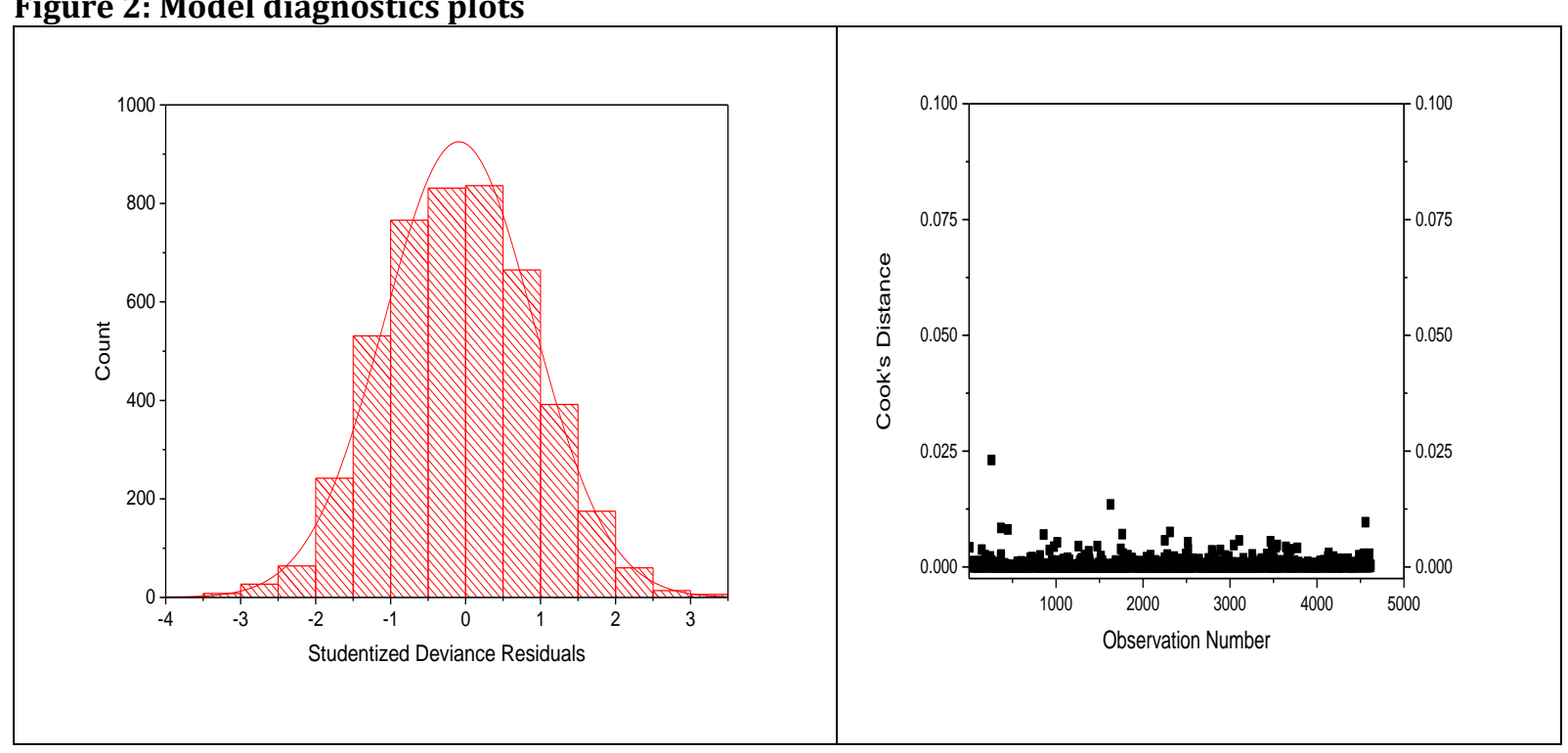

Table 3 shows the effect of the factors on the number of children born by married woman. The results indicate that all the main effects and the five 2-way interactions are significant. The interpretation of the significant main effects which were not involved in the interaction can be interpreted using the rate ratios (RR). All the other main effect variables which are involved in the interaction should be interpreted with special care. For example, because there is a significant interaction effect between media exposure and regional state, the number of children ever born will be influenced by the mother's exposure or non-exposure to mass media but this influence on the number of children ever born will vary from one regional state to another (because of the interaction effect between media exposure and regional state). The interaction plots are useful to assess the effect a pair of factors has on the number of children ever born. An interaction effect is indicated when the lines for different levels of the first factor have unequal slopes. 
Table 3: Analysis of Main and Interaction Effects on the Number of Children Ever Born

\begin{tabular}{lllllll}
\hline Effect & Num DF & Den DF & F Value & Pr $>$ F & Chi-Square & Pr $>$ ChiSq \\
\hline Mother's current age & 1 & 4544 & 44.84 & $<.0001$ & 44.84 & $<.0001$ \\
Residence & 1 & 4544 & 23.45 & $<.0001$ & 23.45 & $<.0001$ \\
Mother's education level & 3 & 4544 & 99.38 & $<.0001$ & 298.14 & $<.0001$ \\
Household head gender & 1 & 4544 & 10.98 & 0.0009 & 10.98 & 0.0009 \\
Household economic status & 2 & 4544 & 9.11 & 0.0001 & 18.21 & 0.0001 \\
Mother's age group at first birth & 2 & 4544 & 94.29 & $<.0001$ & 188.57 & $<.0001$ \\
Media Exposure & 1 & 4544 & 83.30 & $<.0001$ & 83.30 & $<.0001$ \\
Regional State & 10 & 4544 & 2.31 & 0.0105 & 23.11 & 0.0104 \\
Contraceptive & 1 & 4544 & 16.15 & $<.0001$ & 16.15 & $<.0001$ \\
Current age*media exposure & 1 & 4544 & 23.01 & $<.0001$ & 23.01 & $<.0001$ \\
Household head gender *media & 1 & 4544 & 28.78 & $<.0001$ & 28.78 & $<.0001$ \\
Household head gender *Regional & 10 & 4544 & 4.15 & $<.0001$ & 41.46 & $<.0001$ \\
Mother's education*Regional state & 28 & 4544 & 1.70 & 0.0118 & 47.72 & 0.0115 \\
Media exposure *Regional State & 10 & 4544 & 2.92 & 0.0012 & 29.15 & 0.0012 \\
\hline
\end{tabular}

Four factors were not involved in the interaction. Therefore we can interpret the relative ratios of each factor. Table 4 presents the relative ratios and the $95 \%$ confidence intervals of the main effects. If the confidence interval (CI) includes 1, then the result is non-significant and can be interpreted as the mean number of children ever born at the given category equals the mean number of children ever born from the reference category. The rural mother's average number of children ever born is 1.142 times the average number of children ever born from otherwise identical mother who reside in urban. In other words a rural resident mother has $14.2 \%$ more children ever born than otherwise identical urban resident mother. With regard to socio-economic status, the difference in the average number children ever born between the middle and high class mothers' is insignificant. But the difference between the high economic class household mother and the low economic class mothers expected number of children ever born was significant. The low economic class mother has $7.3 \%$ less than the expected number of children of otherwise identical characteristic mother of high economic class. The average number of children ever born from middle class mother is significantly different from that of the low class mother $(\mathrm{RR}=1.072,95 \% \mathrm{CI}(1.032,1.123)$. On the average a mother who gave live birth before the age of 16 years old has $30.7 \%$ more children ever born than otherwise identical mother who started to give live birth in twenties or later age. Likewise, the teenage group member mother at first birth, 16-19, has 24.6\% more children ever born than the mother who gave birth at twenties and above. A non contraceptive user mother has $7.2 \%$ more children ever born than otherwise identical mother who is using contraceptive. 
Table 4: The Poisson Regression Relative Ratios Extracted for Main Effects which are not involved in the interaction

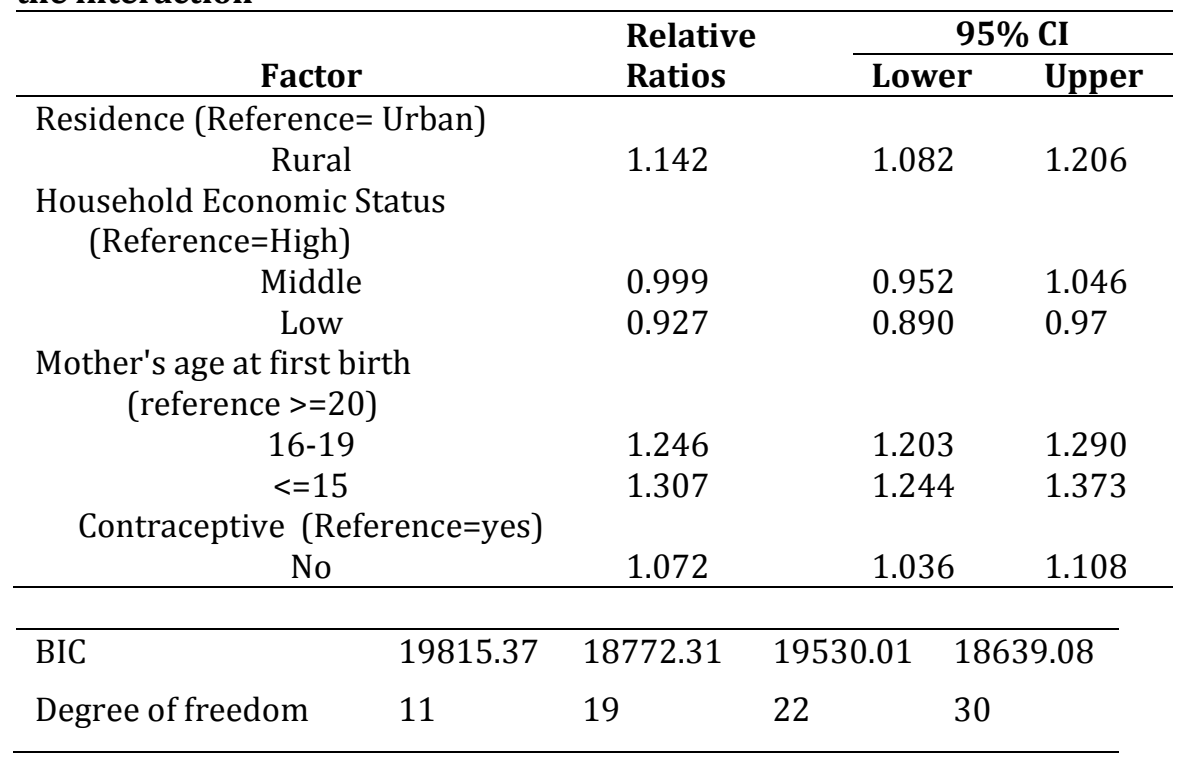

The effect of household head gender on the number of children ever born varies with their media exposure and region (refer Table 3 and Figure3). For the household who has media exposure the headship gender has no significant effect. For the household who has no media access the mother from male household head has significantly more children ever born than that of the mother who head the household. On the other viewpoints, media exposure creates a significant difference for male household heads' effect on the number children ever born, but not for female household heads'. In terms of regions, in Addis Ababa, Dire Dawa, Gambella, SNNP and Somalia regions the difference in the average number of children ever born from female and male household heads is insignificant ( $p>0.065)$. In Afar, Amhara, Harari, Oromiya and Tigray regions female headed household mother, on the average, has significantly lower number of children ever born than a male headed counterpart ( $\mathrm{p}<0.0214)$. Unlike all other regions, in Beneshangul Gumz, female household head mother has more children ever born than otherwise identical mother whose household head is a male $(\mathrm{p}=0.0032)$.

Figure 3: The average number of children ever born by household head gender, media exposure and region

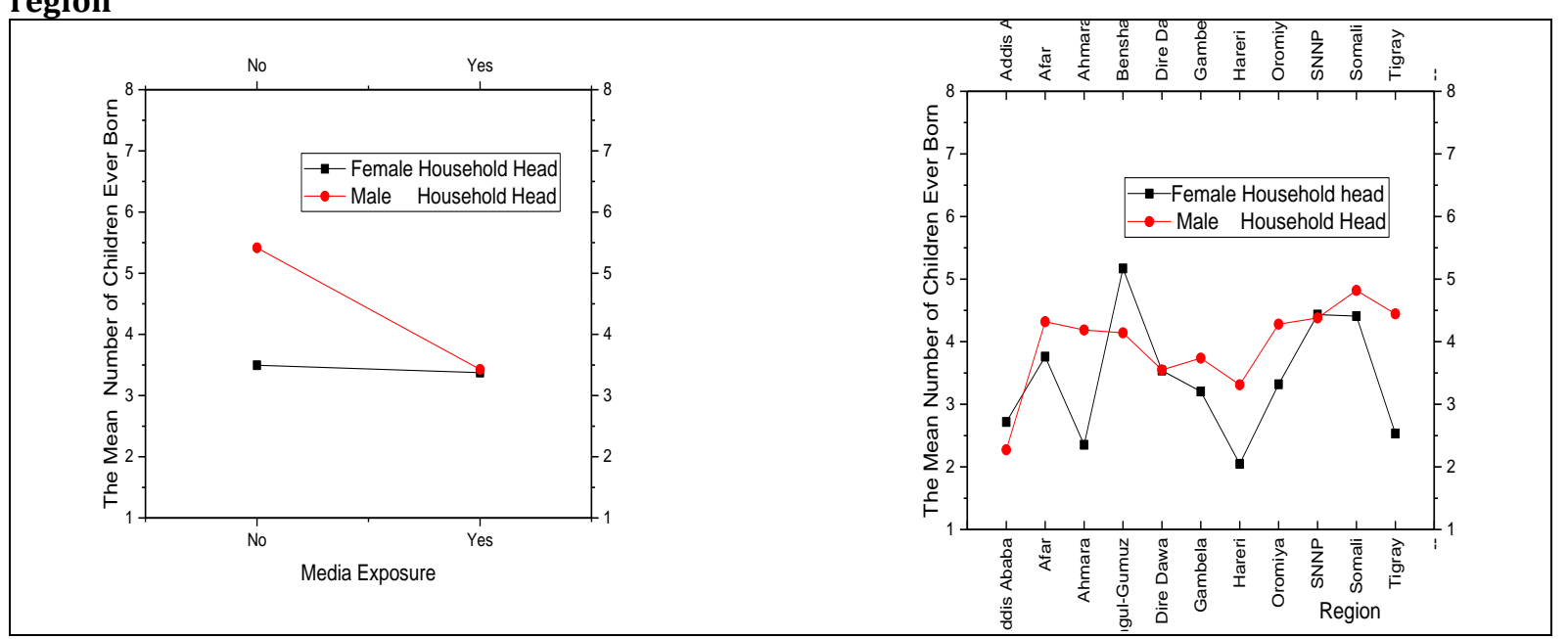

Figure 4 displays the interrelationships between region with media exposure and level of education of mothers. It is noted that the difference between the average number of children ever born for women 
exposed to media and those non-exposed is significant in all regions $(\mathrm{p}<0.001)$. In fact in all regions the average number of children ever born from a woman who has no media exposure is more than from those women who have exposure to media. It can also be noted that generally, the average number of children ever born from a woman decreases as the level of education of mothers gets higher. Exceptional figures belong to Afar and Somali regions where there is no woman with tertiary education. Another exception is Somali mothers with no education have the same average number of children with women having secondary education.

Figure 4: The average number of children ever born by media exposure, education level of the mother and region

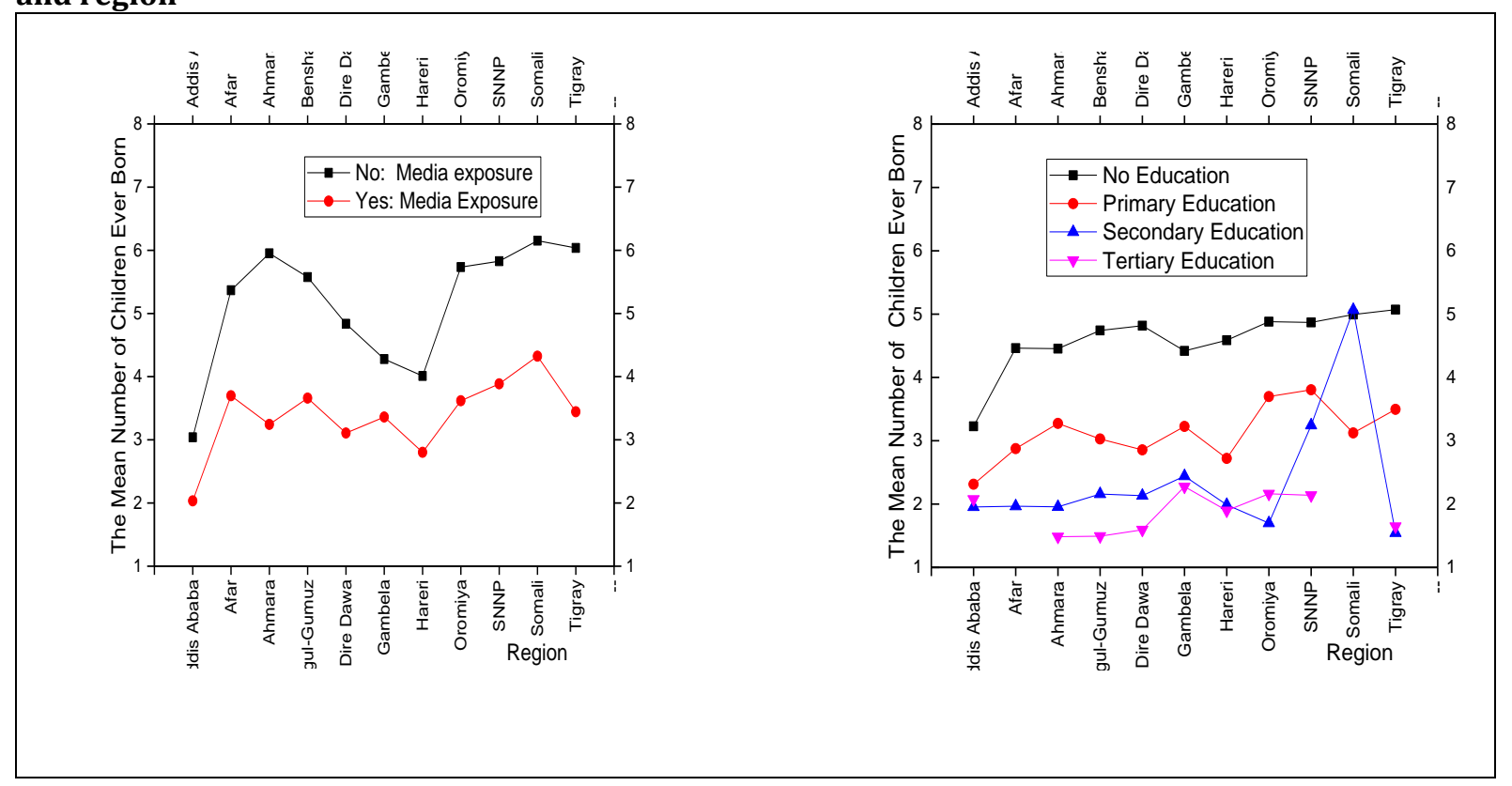

Discussion: In this study we intended to assess the contribution of socioeconomic and demographic determinant factors of fertility among married women in Ethiopia using generalized linear model.The findings of this study showed that place of residence, age at first birth, household economic status and contraceptive use were significant factors affecting the fertility of a woman. Women who live in rural areas give birth to more children than those in urban areas. This is consistent with previous reports from Ethiopia and other developing countries like Nigeria, Ghana, Namibia and Nepal (See for example Hailemariam, 1992; Teklu, Sebhatu, and Gebreselassie, 2013; Gebremedhin and Betre, 2009; Alene and Worku, 2008; Olatoregun et al., 2014; Adhikari, 2010; Indongo and Pazvakawambwa, 2012; Johnson et al., 2011; and Tessema, Zeleke and Ayele, 2013). With respect to contraceptive use, the results showed that women who use contraceptives to control birth had on average less number of children than those who were not using. With regard to the household economic status, the average number of children ever born from a mother of with high economic status is more than from mothers with lower or middle economic status. This is mainly explained by the role children play in the production of crops, and herding cattle and the fact that social capital is measured by the number of children (See also Jara, Dejene and Taha, 2013). It was also found in this study that women who got their first child at earlier ages were more likely to have more children than those women who got at later ages. This may be because the delay in age at first birth has the potential to reduce fertility period. Other studies in Ethiopia by Teklu, Sebhatu and Gebreselassie (2013) and in Namibia by Indongo and Pazvakawambwa (2012) reported findings similar to this study.

The household head gender remains a significant factor on the number of children ever born if the household has no mass media exposure. The result shows women exposed to media are likely to be powerful in making decisions regarding the no of children she should have. For the household who has no media access the male dominance in such decisions was evident. In fact, the deep rooted Ethiopian cultural landscape of gender-role definitions and the modern women empowerment put Addis Ababa, Dire Dawa, Gambella, SNNP and Somalia 
regions which have fair gender equality in household headship. Other regions' result reflects the cultural males' or females' dominance in the household leadership and important decision makings in the family. In most of the regions, the mother's education was a significant factor on fertility and human capital formation. The variation in the regional development imbedded with cultural setups towards girls education some degree of difference is observed among the regions. Nevertheless, in all the regions the less educated the mother the more she tends to have many children.

\section{Conclusion}

Unless Ethiopian population growth is controlled or accompanied by a rapid economic growth it could be a problem to the society. This article was aimed to make a contribution to the study of determinant factors of high fertility using generalized linear model. The capability and novelty of the proposed model was well demonstrated. High fertility was independently associated with residing in urban areas, increased household economic status, younger age at first birth and not using contraceptives. The inclusion of interaction terms to the model greatly expands our understanding of the relationships among the risk factors in the model and drastically changes the interpretation of the main effects. Current age and media exposure, household head gender and media exposure, household head gender and regional state, mother's education and, regional state and media exposure and regional state are jointly associated with fertility status. Government should focus on improving access to media, contraceptives and universal education to young girls and enforcing laws prohibiting early marriage.

Acknowledgement: We thank the Central Statistical Agency (CSA) in Ethiopia for releasing the data used for this study.

Competing interests: The authors declare that they have no competing interests.

Author's contribution: EKM and TMY conceived the study. TMY collected the data. TZ designed and analyzed the data. TZ and EKM wrote and revised the manuscript. All authors read and approved the manuscript.

\section{References}

Adhikari, R. (2010). Demographic, socio-economic, and cultural factors affecting fertility differentials in Nepal. BMC Pregnancy and Childbirth, 10(19).

Agresti, A. (2002). Categorical Data Analysis. 2nd ed. John Wiley \& Sons, Inc., Hoboken, New Jersey.

Alemayehu, T., Haider, J. \& Habte, D. (2010). Determinants of adolescent fertility in Ethiopia. Ethiop. J. Health Dev., 24(1), 30-38.

Alene, G. D. \& Worku, A. (2008). Differentials of fertility in North and South Gondar zones, northwest Ethiopia: A comparative cross-sectional study. BMC Public Health, 8, 397-398.

Allison, P. D. (2001). Logistic Regression Using the SAS System: Theory and Application. John Wiley \& Sons, Inc.

Barron, D. N. (1992). The analysis of count data: over-dispersion and autocorrelation in Sociological Methodology. Peter V. Marsden (ed.) (1992), 179-220: Blackwell.

CSA. (2014). Ethiopian Mini Demographic and Health Survey 2014. Central Statistical Agency, Addis Ababa, Ethiopia.

Falls-Jr, J. A. (2007). Population: A Lively Introduction $5^{\text {th }}$ ed. Washington, DC: Population Reference Bureau, $62(1)$.

Gebremedhin, S. \& Betre, M. (2009). Level and Differentials of Fertility in Awassa Town, Southern Ethiopia. African Journal of Reproductive Health, 13(1), 93-112.

Hailemariam, A. (1992). An Overview of the Determinants of High Fertility in Ethiopia. Ethiopian Journal of Development Research, 14(2), 1-30.

Indongo, N. \& Pazvakawambwa, L. (2012). Determinants of fertility in Namibia. Afr J Reprod Health, 16(4), 5057. 
Jara, D., Dejene, T. \& Taha, M. (2013). Determinants of High Fertility Status among Married Women in Gilgel Gibe Field Research Center of Jimma University, Oromia, Ethiopia: A Case Control Study. Public Health Research, 3(2), 9-17.

Johnson, K., Abderrahim, N. \& Rutstein, S. O. (2011). Changes in the Direct and Indirect Determinants of Fertility in Sub-Saharan Africa. DHS Analytical Studies; No. 23. Calverton, Maryland, USA: ICF Macro.

Lindén, A. \& Mäntyniemi, S. (2011). Using the negative binomial distribution to model over dispersion in ecological count data. Ecology, 92(7), 1414-1421.

McCullagh, P. \& Nelder, J. A. (1989). Generalized linear models. 2nd ed. London: Chapman \& Hall.

Olatoregun, O., Fagbamigbe, A. F., Akinyemi, O. J., Yusuf, O. B. \& Bamgboye, E. A. (2014). A Comparative Analysis of Fertility Differentials in Ghana and Nigeria. Afr J Reprod Health, 18(3), 36-47.

Population Reference Bureau. (2015). World population Data sheet with a special focus on women's empowerment. Washington, DC, Population Reference Bureau.

Teklu, H., Sebhatu, A. \& Gebreselassie, T. (2013). Components of Fertility Change in Ethiopia. Further analysis of the 2000, 2005 and 2011 Demographic and Health Surveys. DHS Further Analysis Reports No.80 Calverton, Maryland, USA: ICF International.

Tessema, G. A., Zeleke, B. M. \& Ayele, T. A. (2013). Birth interval and its predictors among married women in Dabat District, Northwest Ethiopia: A retrospective follow up study. Afr J Reprod Health, 17(2), 39-45.

UN. (2005). Population Challenges and Development Goals 2005. Department of Economic and Social Affairs. New York.

Ver Hoef, J. M. \& Boveng, P. L. (2007). Quasi-Poisson vs. negative binomial regression: how should we model over dispersed count data? Ecology, 88(11), 2766-72.

Zewotir, T. \& North, D. (2015). Analysis of attrition and retention rates using the Generalized Linear Model. South African Statist. Journal, 49(2), 259-271.

Zewotir, T. \& Ramroop, S. (2009). Application of negative binomial regression for assessing public awareness of the health effects of nicotine and cigarettes. African Safety Promotion: A Journal of Injury and Violence Prevention, 7(1), 14-29. 